\title{
Dual Anti-platelet Therapy After Transcatheter Aortic Valve Implantation: Double Trouble?
}

\author{
Faisal Rahman $^{1} \cdot$ Waleed T. Kayani $^{1} \cdot$ Yochai Birnbaum $^{1} \cdot$ Hani Jneid ${ }^{1}$ (D)
}

Accepted: 17 November 2021 / Published online: 22 November 2021

This is a U.S. government work and not under copyright protection in the U.S.; foreign copyright protection may apply 2021

Transcatheter aortic valve implantation (TAVI) has emerged over the last decade as a viable treatment option for patients with severe symptomatic aortic stenosis (AS) across all risk strata with TAVI procedural volumes increasing significantly over time and currently exceeding surgical aortic valve replacement surgery volumes in the USA [1]. The most common complication associated with TAVI is vascular bleeding, which portends a higher mortality risk [2]. Bleeding risk is related to a combination of patient-related factors and pharmacotherapies. Therefore, it is increasingly important to choose, in a shared-decision making process with the patient, the appropriate antithrombotic therapy post-TAVI that will balance bleeding risk with thromboembolic events and long-term valve function to give the best outcomes for patients. This is an important area of consideration as subclinical leaflet thrombosis appears to be more common after TAVI than surgical valve replacement (up to $10-15 \%$ in some series) and is associated with increased risk of cerebrovascular events [3, 4].

In the pivotal PARTNER trials [5], the antithrombotic therapy chosen after TAVI was aspirin and clopidogrel for 6 months, which became the default pharmacotherapeutic approach. However, several randomized controlled trials tried recently to address the issue regarding antithrombotic therapy, with choices including single anti-platelet therapy (SAPT), dual anti-platelet therapy (DAPT), and/or anticoagulation. These investigations parallel the ongoing debate about antithrombotic therapy after percutaneous coronary intervention. The trials of particular note are ARTE (Aspirin versus Aspirin Plus Clopidogrel Following TAVI), GALILEO (Global Study Comparing a Rivaroxaban-Based Antithrombotic Strategy to an Anti-platelet-Based Strategy After Transcatheter Aortic Valve Replacement to Optimize

Hani Jneid

jneid@bcm.edu

1 Section of Cardiology, Department of Medicine, Baylor College of Medicine, Houston, TX, USA
Clinical Outcomes), and the POPular TAVI study. In the smaller ARTE trial of 222 patients [6], aspirin monotherapy - as compared with the combination aspirin and clopidogrel - was associated with significantly lower major or life-threatening bleeding without a difference in death, myocardial infarction, or stroke. This finding was supported by the larger study POPular TAVI trial [7] ( $N=665$ patients), in which the rates of cardiovascular death, stroke, or myocardial infarction were not different between SAPT or DAPT; however, the hazards of major and minor bleeding were significantly higher with DAPT (15.1\% with SAPT vs $26.6 \%$ with DAPT). Interestingly, there were numerically higher cases of symptomatic aortic valve thrombosis with aspirin alone ( 3 patients versus 1 ), but the significance of this finding is unclear given the small numbers. Both studies therefore suggested that aspirin monotherapy after TAVI may be safer and is not associated with a higher risk of adverse outcomes. However, the evidence was far from conclusive given the small sample size and short duration of follow-up in both studies.

The emergence of the serious concern of subclinical leaflet thrombosis and the associated higher risk of cerebrovascular events also led to the investigation of anticoagulation as an alternative therapeutic approach. In the GALILEO [8] trial, patients were randomized to rivaroxaban and aspirin for 4 months followed by rivaroxaban alone or to DAPT for 3 months then aspirin alone. Although rivaroxaban use was associated with lower subclinical leaflet abnormalities, it significantly increased the risk of mortality without decreasing thromboembolic events. This resulted in an early termination of the study. Similarly, results from the ATLANTIS trial investigating apixaban [9] were presented at the American College of Cardiology Scientific Session 2021. In this study, apixaban (5 mg twice daily) was compared to anti-platelet therapy. Like GALILEO, apixaban reduced valve leaflet thrombosis but was associated with higher non-cardiovascular mortality and no benefit in death, MI, or stroke. It wasn't clear why there was no benefit observed 
with respect to clinical outcomes, but in the AUREA trial (presented at TCT 2019 [10]), oral anticoagulation compared to DAPT did not reduce subclinical cerebral lesions on imaging, suggesting that reducing leaflet thrombosis may not necessarily translate into a hard clinical outcome benefit. These studies together did not support the idea of routine use of anticoagulation in TAVI.

In the setting of the aforementioned small studies, Lin et al. [11] in this issue of Cardiovascular Drugs and Therapy reported a meta-analysis of randomized controlled trials comparing aspirin alone versus aspirin plus clopidogrel in patients receiving TAVI. They reported no differences in bleeding (relative risk [RR] 1.22, 95\% confidence interval [CI] 0.62 to 2.39 ) or all-cause mortality rates (RR $1.21 ; 95 \%$ CI 0.52 to 2.84 ) at 30 days but significantly lower risk of bleeding (RR $1.67 ; 95 \%$ CI 1.24 to $2.24, p<0.001$ ) at 6 to 12 months and no difference in the rates of stroke, myocardial infarction, or all-cause mortality in TAVI patients receiving aspirin alone. Their findings support the conclusions drawn from the original smaller studies that SAPT after TAVI reduces bleeding without increasing thrombotic events and mortality.

The meta-analysis by Lin et al. [11] has several strengths including a comprehensive, contemporary, and systematic search; however, it has many noteworthy limitations. The report is an aggregate data meta-analysis and lacks individual patient data. There was no attempt made to search abstracts presented at national proceedings, and considerable heterogeneity in the definitions of bleeding across studies was evident. In addition, the meta-analysis still had moderate power and included a total of 1,086 subjects, reflecting the limitations of the existing evidence. Looking back at the original studies, only a minority of patients who underwent TAVI were randomized in those trials, which may arguably not be representative of the diversity and complexity of current TAVI patients in real-life contemporary clinical practice. Furthermore, these studies have short follow-ups and do not evaluate the long-term effects of the different pharmacotherapeutic strategies on structural valve deterioration. Finally, although SAPT versus DAPT is an important clinical conundrum, the report didn't examine the merits of anticoagulation therapy after TAVI, which - we believe remains a relevant and not-infrequently adopted antithrombotic strategy.

Overall, the findings by Lin et al. [11] underscore the importance of optimizing and individualizing antithrombotic therapy in the increasingly complex TAVI patient, whose bleeding versus thrombotic risk balance is likely to be multifactorial (e.g., related to frailty, other cardiovascular morbidities - such as atrial fibrillation, coronary artery disease, and peripheral arterial disease, and non-cardiovascular comorbidities - such as renal, hepatic, and hematological disease). Bleeding is an important consideration in the management of patients after TAVI, and with improving techniques and device evolution, the risks of complications such as stroke, particularly weeks after the valve deployment, have progressively decreased [1]. Finally, the mechanisms of stroke and bleeding after TAVI is different than with other procedures, such as PCI, and thus, an exact parallel may not be appropriate. Our approach of antithrombotic therapy may require a more investigative approach to figure out how the underlying mechanisms are targeted before rushing to replicate PCI randomized controlled trials [12]. Regardless, the current meta-analysis of the randomized control trials represents an excellent step forward in our understanding of antithrombotic therapy post-TAVI. Reflecting this, the European guidelines recently recommended the use of SAPT, unless there is a compelling indication for DAPT (e.g., coronary stenting) [13]. The American guidelines, on the other hand, recommended the use of aspirin monotherapy as a reasonable strategy (class IIa); however, DAPT for 3 to 6 months if low bleeding risk (class IIb) or vitamin $\mathrm{K}$ antagonist targeting an INR of 2.5 for at least 3 months (class IIb) may be selectively used [14]. A more definitive answer as to the optimal antithrombotic regimen after TAVI may only be possible with further studies with greater power and longer follow-up. All in all, in the era of personalized medicine, it is important to remember that no one size fits all and that clinicians should understand the values and preferences of their patients and continue to engage them in a shared-decision-making process to guide the optimal antithrombotic strategy after TAVI.

\section{Declarations}

Conflict of Interest The authors declare no competing interests.

\section{References}

1. Carroll JD, et al. STS-ACC TVT registry of transcatheter aortic valve replacement. J Am Coll Cardiol. 2020;76(21):2492-516.

2. Zahid S, et al. Trends, predictors, and outcomes of major bleeding after transcatheter aortic valve implantation, from national inpatient sample (2011-2018). Expert Rev Cardiovasc Ther. 2021;19(6):557-63.

3. Chakravarty $\mathrm{T}$, et al. Subclinical leaflet thrombosis in surgical and transcatheter bioprosthetic aortic valves: an observational study. Lancet. 2017;389(10087):2383-92.

4. Makkar RR, et al. Possible subclinical leaflet thrombosis in bioprosthetic aortic valves. N Engl J Med. 2015;373(21):2015-24.

5. Leon MB, et al. Transcatheter aortic-valve implantation for aortic stenosis in patients who cannot undergo surgery. N Engl J Med. 2010;363(17):1597-607.

6. Rodes-Cabau J, et al. Aspirin versus aspirin plus clopidogrel as antithrombotic treatment following transcatheter aortic valve replacement with a balloon-expandable valve: the ARTE (aspirin 
versus aspirin + clopidogrel following transcatheter aortic valve implantation) randomized clinical trial. JACC Cardiovasc Interv. 2017;10(13):1357-65.

7. Brouwer J, et al. Aspirin with or without clopidogrel after transcatheter aortic-valve implantation. $\mathrm{N}$ Engl J Med. 2020;383(15):1447-57.

8. Dangas GD, et al. A controlled trial of rivaroxaban after transcatheter aortic-valve replacement. N Engl J Med. 2020;382(2):120-9.

9. Collet JP, et al. Oral anti-Xa anticoagulation after trans-aortic valve implantation for aortic stenosis: the randomized ATLANTIS trial. Am Heart J. 2018;200:44-50.

10. ClinicalTrials.gov. Dual antiplatelet therapy versus oral anticoagulation for a short time to prevent cerebral embolism after TAVI (AUREA) Available online: https://clinicaltrials.gov/ct2/show/ NCT01642134.

11. Lin X. Aspirin alone versus dual antiplatelet therapy after transcatheter aortic valve replacement: a systematic review and metaanalysis. Cardiovasc Drugs Ther 2021:In press

12. Dauerman HL, et al. Prolonged clotting time among patients undergoing transcatheter aortic valve replacement. J Am Coll Cardiol. 2019;74(6):820-1.
13. Ten Berg J, et al. Management of antithrombotic therapy in patients undergoing transcatheter aortic valve implantation: a consensus document of the ESC Working Group on Thrombosis and the European Association of Percutaneous Cardiovascular Interventions (EAPCI), in collaboration with the ESC Council on Valvular Heart Disease. Eur Heart J. 2021;42(23):2265-9.

14. Writing Committee M, et al. 2020 ACC/AHA guideline for the management of patients with valvular heart disease: executive summary: a report of the American College of Cardiology/ American Heart Association Joint Committee on Clinical Practice Guidelines. J Am Coll Cardiol. 2020;77(4):450-500.

Publisher's Note Springer Nature remains neutral with regard to jurisdictional claims in published maps and institutional affiliations. 